- Article type: paper

- Date text written or revised: September 2014

- Number of words in your main text and tables: 4900

- Number of figures: 7

\title{
Application of Network Traffic Flow Model for Road Maintenance
}

Author 1

- Chao Yang, PhD

- Nottingham Transportation Engineering Centre, University of Nottingham, Nottingham, UK Author 2

- Rasa Remenyte-Prescott, $\mathrm{PhD}$

- Nottingham Transportation Engineering Centre, University of Nottingham, Nottingham, UK Author 3

- John Andrews, PhD

- Nottingham Transportation Engineering Centre, University of Nottingham, Nottingham, UK

\section{Full contact details of corresponding author.}

Dr Rasa Remenyte-Prescott

Nottingham Transportation Engineering Centre

University of Nottingham

University Park

Nottingham NG7 2RD UK

$\mathrm{t}:+44(0) 1158467366$

f: $+44(0) 1159513909$

e: r.remenyte-prescott@nottingham.ac.uk 


\section{Abstract}

This paper shows how the evolution of two-way traffic flows on a local highway network can be predicted over time, based on a network level traffic flow model (NTFM) that is used to model both urban and motorway road networks. After a brief review of the main principles of NTFM and its associated sub-models, the paper describes how a maintenance worksite can be modelled using a roadwork node sub-model and a network solution routine in NTFM. In order to model the two-way traffic flow in the road network, an iterative simulation method is utilised to generate the evolution of dependent traffic flows and queues. NTFM has been applied to model the traffic characteristics and effects of maintenance activities on the local Loughborough-Nottingham highway network. It was demonstrated that the methodology is useful in selecting various worksite arrangements in order to reduce maintenance effects on road users.

\section{Keywords chosen from ICE Publishing list}

traffic engineering; road maintenance; mathematical modelling

\section{List of notation}

G : Green phase for traffic in the direction of roadworks

R : Red phase for traffic in the direction of roadworks

$A_{m} \quad$ : Amber phase for traffic in the direction of roadworks

Cy : Cycle time for traffic

C : The flow capacity

S : Saturation flow

сp : : Link capacitance

$\mathrm{L} \quad$ : Length of the link

$\mathrm{N} \quad$ : Number of lanes on the link 


\section{Introduction}

During the past decade the transportation infrastructure, particularly the highway network, in the UK has deteriorated significantly due to the increasing number of road users. Audit Scotland (Audit Scotland, 2011) published that the condition of Scotland's roads had worsened over the past six years, with only $63 \%$ of roads in an acceptable condition, and the level of the maintenance backlog had more than doubled since 2004 to reach £2.24 billion. In addition, the travel delay cost to road users usually exceeds the cost of maintenance. In order to minimise travel delays experienced on the highway network, especially when roadworks are in progress, modelling techniques, such as NTFM, can be employed to facilitate a better decision making process on roadworks, considering not only maintenance costs but also traffic delays (Yang et al., 2013). NTFM forecasts the traffic flow rates and queue dynamics in the highway network. Also, it has the capability to reduce the flow rate capacity on parts of the network when maintenance work is conducted, making it a suitable platform for evaluating travel delays. By comparing the traffic outputs and queues in the network under alternative maintenance strategies, the best way of maintaining the serviceability level of highways can be found.

Many macroscopic mathematical models have been formulated to describe traffic dynamics on urban roads and motorways. Lighthill and Whitham (Lighthill and Whitham, 1955) and Richards (Richards, 1956) developed a macroscopic, hydrodynamic model (LWR) based on first order differential equation to describe traffic behaviour for a single one-way road. Payne (Payne, 1971) provided a second-order model by further considering the driver's reaction time that results in a dynamic mean speed equation. As an extension to the LWR model, Daganzo (Daganzo, 1994) built a cell transmission model (CTM) to evaluate the traffic on a highway network with a single entrance and exit. Some improvements of CTM are further explained in literature (Lo, 1999; Lo et al., 2001; Daganzo, 1995). However, CTM is only sufficient for signalised networks where the traffic interaction among flows from competing arms is eliminated. Messmer and Papageorgiou (Messmer and Papageorgiou, 1990) developed a macroscopic simulation program, METANET, based on the second order model in order to investigate the traffic for motorway networks. Further research has been carried out to develop METANET (Deflorio, 2003; Breton et al., 2002; Hegyi et al., 2005, Karimi et al., 2004, 
Van den Berg et al., 2007). One feature of METANET is that the inflow and outflow for each node is characterised by turning ratios of traffic flows from each link that is connected to the node, as a result the effects of conflicting requirements of the traffic flows from competing directions at priority junctions are not considered. Furthermore, gap acceptance theory and queuing theory have been extensively applied to investigate the traffic at different junctions (Brilon et al., 1999; Cowan, 1975; Daganzo, 1977; Kremser, 1962; Kremser 1964; Ning, 2001; Plank and Catchpole, 1984; Siegloch, 1973; Tanner, 1962; Troutbeck and Brilon, 1997; Guo and Lin, 2011; Doan and Ukkusuri, 2012; Weng and Meng, 2013; Ma et al., 2013; Carey et al., 2014). However, some of the models can become inefficient when modelling directional flow and identifying traffic behaviours at network level (Robinson et al., 1999; Ruskin and Wang, 2002; Tian et al., 2000; Tracz and Gondek, 2000; Chen and Kasikitwiwat, 2011). In order to overcome some of these deficiencies, the NTFM has been formulated by the authors to include an increased range of junction types, such as signalised junctions and priority junctions, and to predict the traffic flow rates, queues and travel delays at network level.

Using the NTFM road network is evaluated based on the principle of a maximum capacity flow rate at junctions with competing flows. One feature of this model is that it takes advantage of the observed traffic flow turning ratios at junctions rather than an O-D (origin-destination) trip matrix, as such data is more easy to obtain than the data on individual journey plans. Furthermore, two-way traffic is studied in NTFM by identifying the relationships of dependent traffic flows and queues in the network using an iterative method. The novelty of this paper is that a roadwork node sub-model is introduced in the NTFM, which is then applied to evaluate the effect of maintenance activities on network level performance. Therefore, NTFM can be further used to optimise road maintenance, for example, find the best maintenance schedule, considering the start and the end of roadworks during the day, or find the best worksite arrangements, considering traffic signal phases through roadworks. A roadwork model is described in section 2, and the methodology is illustrated by the NTFM application on the local Loughborough-Nottingham highway network under different maintenance scenarios, described in section 3 . Final remarks about the modelling technique and future work are given in section 4 . 


\section{Overview of the Road Network Model}

In NTFM the network is modelled in terms of links, representing roads, and nodes between them, representing junctions, briefly described below.

\subsection{Sub-models in NTFM}

Traffic flow complexity is introduced through the use of junction sub-models, when separate lanes are modelled on the road links and the flow capacity and the capacitance on each lane are considered, instead of using shared lanes for mixed traffic, considered in previous research. Note, that the flow capacity is the number of vehicles passing the link, expressed in terms of passenger car unit (pcu) per hour, and the link capacitance is the maximum number of cars which can queue on the link. Such models are constructed to reproduce the traffic movement at different junctions, as listed in Table 1. A brief overview of modeling assumptions for different types of junctions is given below. For example, one feature of signalised junctions is that the interactions among traffic flows from competing directions are removed by traffic control. While modelling priority junctions, the evaluation of traffic is based on right-of-way rules, where the entering traffic flow for each arm of the junction is influenced by the flow capacity and the conflicting traffic flows from competing arms. For one-way junctions, except the on-ramp, traffic entering is only restricted by the corresponding flow capacity, and on-ramp is evaluated as a priority junction, since the traffic on the slip road must allow the traffic on the major road to pass before it can join the major road.

Table 1 (Junction types in NTFM) to be inserted here.

\subsubsection{Roadwork node model}

The implementation of maintenance work on a road link can be represented by limiting the exiting traffic flow capacity of this link. The length and location of worksite, that are used to define the geometry of worksite, also need to be considered, as does the link capacitance which decreases during maintenance. Therefore in NTFM a special roadwork node is employed to represent the part of road link under maintenance, which specifies the location of worksite, the length of the worksite, and the number of lanes in service. A typical roadwork node is shown in Figure 1, where:

A : Downstream junction 
B : Upstream junction

C : Roadwork node

$\mathrm{x} \quad$ : The distance between downstream junction and worksite

$m_{1}, m_{2}:$ The length of worksite and the length of the remaining ling (without maintenance)

$L \quad$ : The length of link $A B$

$n_{1}, n_{2}:$ the number of lanes remaining open at the worksite and the rest of the link: in Figure $1 n_{1}=1$ and $\mathrm{n}_{2}=2$.

Figure 1 (A road link in maintenance) to be inserted here.

Roadworks usually cause traffic bottlenecks, when the flow capacity is reduced, and such situations can be represented by the roadwork node in the network. For example, the flow capacity of link $B A$ is mainly characterised by its weakest point $C$. The values of default link capacity associated with the type of road are shown in Table 2 (DfT, 2004), which are used in the calculations of travel delays.

Table 2 (Default road capacities) to be inserted here.

\subsubsection{Single carriageway maintenance}

On single carriageway roads, a shuttle working arrangement is applied to assign gaps for the two competing flows. At worksites with shuttle working each direction suffers intermittent intervals of flow passing the site, alternating with intervals of zero capacity. The layout of the worksite with shuttle working is illustrated in Figure 2. Traffic lights are applied to assign the gap for each directional flow, and the cycle time $C_{y}$ is represented by:

$$
C_{y}=G+R+A_{m}
$$

Figure 2 (Single carriageway road with shuttle working) to be inserted here.

where

G : Green phase for traffic in the direction with roadworks

R : Red phase for traffic in the direction with roadworks

$A_{m} \quad$ : Amber phase, that represents the time for the vehicles already flowing through the worksite to exit, which is proportional to the length of worksite

The site capacity for traffic flow in the direction with roadworks is represented by the formula: 


$$
C_{w}=\frac{S \cdot G}{C_{y}}
$$

where $C_{w}$ is the flow capacity at roadworks and $S$ is the saturation flow (pcu/hr). The default value of saturation flow in QUADRO is $1800 \mathrm{pcu} / \mathrm{hr}$ (DfT, 2004), which has also been used in this study. Conversely, the site capacity for the other direction is calculated as, expressed as the capacity $C_{0}$ :

$$
C_{o}=\frac{S \cdot R}{C_{y}}
$$

\subsubsection{Multi-lane carriageway maintenance}

For a multi-lane carriageway, more than one lane can be maintained. If $N$ lanes are in service through the worksite each with an individual normal capacity $C_{n}$, then the worksite capacity $C_{w, N}$ is formulated, as shown below (DfT, 2004):

$$
C_{w, N}=0.85 \times N \times C_{n}
$$

The default value of $C_{n}$ is shown in Table 2. The evaluation of the traffic flow through the worksite follows the rules of NTFM calculation process, given in the next section.

\subsection{Network Solution Routine}

There are two main steps in the evaluation of traffic condition for a highway network. Firstly, the traffic inputs and outputs for each junction in the network are calculated iteratively until they remain unchanged by further iterations. Afterwards, the portion of any queue that exceeds the link capacitance propagates back to its upstream links. As a consequence, the traffic condition for the network at the current time step is achieved, and it is passed to the next time step as the initial traffic condition. Based on this rule, the traffic condition state for a highway network over the forecasting span is evaluated.

\section{Model Application and Numerical Performance}

To illustrate the performance of NTFM a case study based on the Loughborough-Nottingham highway network is presented. The highway network includes both urban road links and motorway links. In addition to normal road travel conditions, roadworks are introduced to demonstrate the effect of maintenance activities on network performance. This process demonstrates the changes of traffic flow through the highway network under different scenarios. 


\subsection{Loughborough-Nottingham Highway Network}

This network is composed of trunk roads and certain parts of the regional rural and urban road network. An overview of the highway network is shown in Figure 3. The central framework of the highway network is composed of trunk roads that are recognised as the boundary of the highway network. There are three main routes from Loughborough to Nottingham, which are A60-A52, A6-A453-A52 and A512-M1-A52.

Figure 3 (Loughborough-Nottingham highway network) to be inserted here.

For simplicity, only trunk roads and roads that connect to major junctions are retained in the network. Some symbols, such as M1 and A52, denote the road class and road number of the road links; while other symbols represent the type and ID of junctions, for instance, the "diverge" junction in Loughborough is named D2.

There are 47 junctions modelled in the network, including 8 roundabouts $(R), 3$ signalised roundabouts (SR), 5 diverge junctions (D), 3 merge junctions (MG), 12 off-ramps and 12 on-ramps (both denoted by S), 2 signalised T-junctions (ST), and 2 signalised intersections (SI). These junctions are evaluated using a chosen sequence. In addition, roadwork nodes ( $R N)$ have been introduced as appropriate to investigate the influence of maintenance activities to the traffic in the network. As such road links provided in the network depend on the local road classes, they need to be addressed differently according to the road class of the link it belongs to. In this case study three RNs are added to the network, which are RN1 (dual 2-lane carriageway), RNS1 (single carriageway) and RNM1 (motorway).

\subsection{Network Calibration}

The model calibration procedure enables the whole network to represent the traffic conditions with sufficient accuracy. There are 43 links in the modelled highway network, including a range of types of roads. The flow capacity for each link is determined as defined in (DfT, 2004), and the capacitance for each link is calculated as:

$$
c p=\frac{L \times N}{\text { headway }}
$$


where $c p(\mathrm{pcu})$ is the link capacitance, and $L(\mathrm{~km})$ and $N$ is the length of the link and the number of lanes on the link, respectively. Headway is defined as $7 \mathrm{~m}$ in NTFM, including vehicle length and the gap between two adjacent vehicles (Cowan 1975).

The available information on this highway network for model calibration was a set of traffic counts which had been collected at various locations on trunk roads and at various junctions over the recent few years, including entering flows and turning movements for monitored junctions. Specifically, the traffic data used in this study was obtained from the Highways Agency (HA, 2011) and Nottingham County Council, and applied to the traffic flow model in the form of two-way hourly traffic flow. The characteristics of the traffic data are illustrated in Table 3.

Table 3 (Characteristics of traffic data) to be inserted here.

Table 4 and Table 5 give an illustration of data used in this study. It contains the number of passenger car units per hour (pcu/h) that enter a junction and take relevant turnings after it (L-left, A-ahead, R-right). Such data is given for each one-hour interval throughout a day. This value in each cell is an average of the variable calculated over the period of data collection. For brevity, one example of each major junction type on the selected network has been presented, i.e. Table 4 contains data for a signalised intersection (SI2), an off-ramp junction (S9) and a signalised roundabout (SR1), and Table 5 contains data for a diverge junction (D1), signalised T-junction (ST1) and a roundabout (R1).

Table 4 (Example 1 of traffic data) to be inserted here.

Table 5 (Example 2 of traffic data) to be inserted here.

\subsection{Highway network performance under normal conditions}

The focus of the analysis is to predict the outflow and queue length for each junction/link in the highway network and to identify the weak links/junctions that experienced severe traffic congestion. The model is executed from 7:00 am to 11:00 pm using a one-hour time step. On the basis of the results obtained from modelling the whole network, it can be concluded that junctions R1, R6 and R7 suffered traffic congestion during the morning and afternoon peak periods, while other junctions can accommodate their entering flows without disturbance, as summarised in Table 6. 
Table 6 (Traffic condition states for the congested junctions/links in the example network) to be inserted here. Note, that the traffic condition of a road link after the analysis is carried out is also distinguished: "NQ" means no travel delay occurred; road link that represented by "MINQ" indicates that only a few vehicles, under the threshold of 100 vehicles, are delayed; when there are more than 100 vehicles queuing on the link is represented by "MAJQ". The weakest links of the network are identified. For example during the period 7:008:00, a relatively small amount of traffic is present on the highway network, therefore, vehicles can travel without any delays. At the second time step 8:00-9:00, there are more commuters in the network heading to work/school locations, which results in heavy traffic congestions at some large junctions. For instance, the priority junction R6 assigns more transition capacity to the major routes that experience higher traffic. The two arms of R6 on A52 are used as major routes, and some vehicles on the minor arm from B5010, have to queue, since the amount of the traffic passing by this arm exceeds the flow capacity. In the next time step, some queues have dissipated, such as at junction R7, and some remained to cause travel delays, such as at R1. Eventually, all the queues on the network dissipate throughout the morning and further delays are experienced during afternoon rush hour, as commonly observed on this network. Queues on the network are illustrated in Figure 4. The queue of the network is calculated as the sum of all the queues formed in the network at each time step. It can be seen that the highway network experienced heavy traffic congestion during the rush hours. Figure 4 (Traffic condition of the example network under normal condition) to be inserted here.

\subsection{Performance of highway network due to maintenance}

The ability of NTFM to model maintenance on the three types of road is illustrated below.

\subsubsection{Dual 2-lane carriageway maintenance}

To investigate the effect of maintenance activities on a dual 2-lane carriageway, RN1 node is assigned to link MG2-R4. There are three cases considered for RN1 in terms of the worksite parameters defined in Section 2.1.1, as shown in Table 7.

Table 7 (Road geometries for roadworks on dual 2-lane carriageway) to be inserted here.

Case 1 represents network conditions without maintenance, while for Case 2 and Case 3 which represent network conditions with maintenance and the length of the worksite in Case 3 is twice of that in Case 2. The 
traffic characteristics of the network through the day in each case are illustrated in Figure 5 , the graph on the left refers to the total flow of the highway network throughout the day and the graph on the right presents travel delay occurred in the network.

Figure 5 (Highway network with roadworks on the dual 2-lane carriageway: (a) Exiting traffic flow through the network; (b) Total queue within the network) to be inserted here.

As only one lane of the dual carriageway is open due to maintenance (Case 2 and Case 3), longer queues than in Case 1 occur, especially during the afternoon peak period when the traffic is heavy. Due to maintenance the road capacity on RN1 is significantly reduced. As a consequence, the queue on link MG2-R4 with roadworks exceeded the corresponding link capacitance and further effects were observed on the links related to MG2-R4. No noticeable differences of network performance were observed between Case 2 and Case 3, since the capacitance of the link with roadworks is very similar between the two cases. A range of other worksite arrangements can be implemented using NTFM.

\subsubsection{Single carriageway maintenance}

A single carriageway SR2-R4 is analysed in this illustration and the worksite is defined by RNS1 node. Three maintenance scenarios are investigated at RNS1, described in Table 8.

Table 8 (Road geometries for roadworks on the single carriageway) to be inserted here.

When RNS1 is undergoing repair, the traffic signal control is employed to assign gaps for each directional flow, and the green splits for both directions are assumed to be equal. For Case 2 the green splits for both directions are defined as $45 \%$ with the amber phase taking $10 \%$ of the whole cycle, while for Case 3 the green splits are $40 \%$ and the amber phase is $20 \%$ (due to the length of worksite being double of that in Case 2 ).

The traffic conditions of the whole network under normal condition and that with the repair at RNS1 are compared in Figure 6. Due to maintenance the flow on the single carriageway is restricted by the opposing flow, and queues occur. In addition, Figure 6 shows that the traffic delay experienced in the network in Case 3 is more than twice of that in Case 2, as the flow restrictions due to the longer worksite and smaller green splits in Case 3 have more effects on the network than in Case 2. 
Figure 6 (Highway network with roadworks on the single carriageway: (a) Exiting traffic flow through the network; (b) Total queue within the network) to be inserted here.

\subsubsection{Motorway maintenance}

Link S4-S7 is a typical 3-lane motorway, on which maintenance is assumed to be performed at RNM1. The parameters of this link, as described in Figure 1, are defined as: $x=4 \mathrm{~km}, m_{1}=1.12 \mathrm{~km}, m_{2}=3.2 \mathrm{~km}, L=8.32 \mathrm{~km}$. There are two cases considered for RNM1: in Case 1 no maintenance is carried out on the three lane motorway and in Case 2 one lane is closed for maintenance. The performance of the network is illustrated in Figure 7. Travel delays on the motorway only occurred in rush hours and hardly any additional delays were experienced due to maintenance in Case 2. In this illustration, the flow capacity on the motorway is relatively large in comparison to the entering traffic flow, and the loss of one service lane causes minor delays only.

Figure 7 (Highway network with roadworks on the motorway: (a) Exiting traffic flow through the network; (b) total queue within the network) to be inserted here.

\subsubsection{Discussion}

Based on the results in Sections 3.3 and 3.4, it can be concluded that the model gives realistic results, i.e. in most cases travel delays occurring on the network during maintenance are more severe than those under normal conditions, since the flow capacity of the link under maintenance is reduced due to lane closure and causing traffic bottlenecks. Moreover, it was found that the network with maintenance at a single carriageway caused the most severe traffic congestion in the network, due to the fact that the single lane has to be used by the opposing traffic, controlled by signals, as shown in Figure 6 . The least severe traffic congestion was caused by maintenance on the motorway, especially when the entering flow did not reach the road capacity, as shown in Figure 7.

As observed in practice, road maintenance has a great impact on the network performance, especially when the flow exceeds the capacity of the network even in the situations without roadworks, i.e. at morning and afternoon peaks, as demonstrated in Table 4. Thus, when making worksite arrangements, the expected flow rates and flow capacities through the day should be considered in order to minimise travel delays and reduce user costs. Furthermore, the variation in the length of the worksite and traffic control signals, especially during 
single carriageway maintenance, described in Table 6, play an important role, as demonstrated in Figure 7. Therefore, appropriately chosen parameters, such as appropriate phases of traffic signals at maintenance site, can also cause less disruption.

The modelling results illustrate how highway networks are affected by maintenance works, especially when the flow along a road at peak times is a lot higher than the road capacity. By analysing the travel delay incurred by maintenance, roadworks can be planned to cause less disruption when possible, i.e. perform maintenance when the traffic is low or implement breaks during maintenance hours to avoid the peak times of traffic. Since maintenance effects on the network can be modelled using the NTFM, the results can be used as an input to an optimisation routine used to find the best maintenance arrangements for the network. However, additional factors, such as the increased cost of maintenance at night, also need to be considered while carrying out maintenance planning. The best solutions can be found when considering the two conflicting stakeholders - road users and highway agency, since carrying out road works at night can be convenient for road users but expensive and impractical for highway agency.

\section{Conclusions}

This paper presents the network traffic flow model (NTFM) which is used to calculate traffic flows in the network, especially when additional delays on the network are caused by road maintenance. The main novel features of the NTFM are the ability:

i. To account for both motorway and urban roads in the same model, as illustrated in Figure 3, reflecting the interactive nature of the types of systems, commonly modelled separately.

ii. To model two-way traffic flow by employing iterative simulation method in order to express dependent traffic flows round the network, as described in Section 2.2 .

iii. To describe and compare maintenance arrangements on different types of road by introducing a roadwork node sub-model in the network, as illustrated in Section 3.4.

The paper focusses on a case study, which has been presented in order to illustrate how traffic conditions on the local Loughborough-Nottingham highway network can be investigated. Traffic flows and queues throughout the day on the network were calculated and the results demonstrated that the delays on the network increase significantly when maintenance is implemented. In addition, the effects of different maintenance arrangements to the network, such as traffic signal phases through roadworks and number of lanes in operation, described in Tables 5 and 6, can be compared. 
NTFM is a useful tool for evaluating the traffic condition of a road network in terms of vehicle outflows and traffic queues and to demonstrate disturbances on the network due to maintenance activities on different types of roads, as shown in Figures 5, 6 and 7. This tool could be used to predict travel delays at network level that occur due to maintenance and to suggest optimal maintenance arrangements in order to minimise the delays. Further research could be carried out in using NTFM as an input to optimisation of maintenance schedule taking into account the effects of delays caused by different start time of roadworks during the day. Another possible direction is to develop an algorithm for traffic rerouting, so that delays at heavily congested areas can be minimised and flow is distributed to local links on the network to avoid further delays.

\section{ACKNOWLEDGMENT}

John Andrews is the Royal Academy of Engineering and Network Rail Professor of Infrastructure Asset Management. He is also Director of The Lloyd's Register Foundation* Centre for Risk and Reliability Engineering at the University of Nottingham. Rasa Remenyte-Prescott is the Lloyd's Register Foundation Lecturer in Risk and Reliability Engineering. They would both like to express their gratitude to all of these organisations for their support.

*The Lloyd's Register Foundation (The LRF) supports the advancement of engineering-related education, and funds research and development that enhances safety of life at sea, on land and in the air.

\section{References}

Audit Scotland (2011). Audit Scotland Annual Report.

Breton P, Hegyi A, De Schutter B and Hellendoorn H (2002). Shock wave elimination/reduction by optimal coordination of variable speed limits. In Proceedings of the IEEE 5th International Conference, Intelligent Transportation Systems, Singapore, 3-6 September: 225-230.

Brilon W, Koenig R and Troutbeck RJ (1999). Useful estimation procedures for critical gaps. Transportation Research Part A: Policy and Practice 33(3-4): 161-186.

Carey M, Humphreys P, McHugh M and Mclvor R (2014). Extending travel-time based models for dynamic network loading and assignment, to achieve adherence to first-in-first-out and link capacities. Transportation Research Part B: Methodological 65: 90-104. 
Chen A and Kasikitwiwat P (2011). Modelling capacity flexibility of transportation networks. Transportation Research Part A: Policy and Practice 45(2): 105-117.

Cowan RJ (1975). Useful headway models. Transportation Research 9(6): 371-375.

Daganzo CF (1977). Traffic delay at unsignalized intersections: Clarification of some issues. Transportation Science 11(2): 180.

Daganzo CF (1994). The cell transmission model - a dynamic representation of highway traffic consistent with the hydrodynamic theory. Transportation Research Part B—Methodological 28(4): 269-287.

Daganzo CF (1995). The cell transmission model.2. Network traffic. Transportation Research Part BMethodological 29(2): 79-93.

Deflorio FP (2003). Evaluation of a reactive dynamic route guidance strategy. Transportation Research Part C: Emerging Technologies 11(5): 375-388.

DfT (2004). Design manual for roads and bridges (DMRB). Economic Assessment of Road Maintenance. 14. Department of Transport

Doan K and Ukkusuri SV (2012). On the holding-back problem in the cell transmission based dynamic traffic assignment models. Transportation Research Part B: Methodological 46(9): 1218-1238.

Guo RJ and Lin BL (2011). Gap acceptance at priority-controlled intersections. Journal of Transportation Engineering-ASCE 137(4): 269-276.

HA (2011). Traffic Information Database. Highways Agency.

Hegyi A, Bart De S and Hellendoorn J (2005). Optimal coordination of variable speed limits to suppress shock waves. Intelligent Transportation Systems, IEEE Transactions 6(1): 102-112.

Karimi A, Hegyi A, De Schutter B, Hellendoorn H and Middelham F (2004). Integration of dynamic route guidance and freeway ramp metering using model predictive control. In Proceedings of the American Control Conference, Boston, June 30-July 2: 5533-5538.

Kremser H (1962). Ein Zusammengesetztes Wartezeitproblem Bei Poissonschen Verkehrsströmen (A complex problem of delay with Poissondistributed traffic flows). Osterreichisches Ingenieur-Archiv 16: 231252.

Kremser H (1964). Wartezeiten Und Warteschlangen Bei Einfadelung Eines Poissonprozesses in Einen Anderen Solchen Prozess (Delays and queues with one Poisson process merging into another one). Österreichisches Ingenieur-Archiv 18: 269-274.

Lighthill MJ and Whitham GB (1955). On kinematic waves. II. A theory of traffic flow on long crowded roads. Proceedings of the Royal Society of London. Series A. Mathematical and Physical Sciences 229(1178): 317-345.

Lo HK (1999). A novel traffic signal control formulation. Transportation Research Part A: Policy and Practice 33(6): 433-448.

Lo HK, Chang E and Chan YC (2001). Dynamic network traffic control. Transportation Research Part A: Policy and Practice 35(8): 721-744.

Ma W, Liu Y, Head L and Yang X (2013). Integrated optimisation of lane markings and timings for signalised roundabouts. Transportation Research Part C: Emerging Technologies 36: 307-323. 
Messmer A and Papageorgiou M (1990). METANET: A macroscopic simulation program for motorway networks. Traffic Engineering and Control 31(9): 466-470.

Ning W (2001). A universal procedure for capacity determination at unsignalized (priority-controlled) intersections. Transportation Research Part B: Methodological 35(6): 593-623.

Payne HJ (1971). Models of freeway traffic and control. Bekey GA (ed). Mathematical Models of Public Systems, Simulation Council Proceedings Series, La Jolla, California, 1(1): 51-61.

Plank AW and Catchpole EA (1984). A general capacity formula for uncontrolled intersection. Traffic Engineering Control 25(6): 327-329.

Richards PI (1956). Shock waves on the highway. Operations Research 4(1): 42-51.

Robinson BW et al (1999). Extensions of theoretical capacity models to account for special conditions.

Transportation Research Part A: Policy and Practice 33(3-4): 217-236.

Ruskin HJ and Wang R (2002). Modeling traffic flow at an urban unsignalized intersection. Proceedings of the International Conference on Computational Science-Part I, Springer-Verlag, Amsterdam, The Netherlands, April 21-24: 381-390.

Siegloch W (1973). Die Leistungsermittlung an Knotenpunkten ohne Lichtsignalsteuerung (Capacity determination at intersections without traffic signals). Schriftenreihe Strabenbau und strabenverkehrstechnik. Vol. 154.

Tanner JC (1962). A theoretical analysis of delays at an uncontrolled intersection. Biometrika 49(1/2): 163170.

Tian ZZ et al (2000). A further investigation on critical gap and follow-up time. Transportation Research Circular (E-C018): 4th International Symposium on Highway Capacity, Hawaii, June 27-July 1: 397-408. Tracz M and Gondek S (2000). Use of simulation to analysis of impedance impact at unsignalized intersections. Transportation Research Circular (E-C018): 4th International Symposium on Highway Capacity, Hawaii, June 27-July 1: 471-484.

Troutbeck RJ and Brilon W (1997). Unsignalized intersection theory. In: Revised Monograph on Traffic Flow Theory, Chapter 8, Publication by Transportation Research Board: 1-47, http://www.fhwa.dot .gov/publications/research/operations/ttt/chap8.pdf. Van den Berg M, Hegyi A and De Schutter B (2007). Integrated traffic control for mixed urban and freeway networks: A model predictive control approach. European Journal of Transportation and Infrastructure Research 3(3): 223-250.

Weng J and Meng Q (2013). Estimating capacity and traffic delay in work zones: an overview. Transportation Research Part C: Emerging Technologies 35: 34-45.

Yang C, Remenyte-Prescott R and Andrews JD (2013). A network traffic flow model for motorway and urban highways. Journal of the Operational Research Society, advance online publication 


\section{Figure captions}

Figure 1. A road link in maintenance

Figure 2. Single carriageway road with shuttle working

Figure 3: Loughborough-Nottingham highway network

Figure 4: Traffic condition of the example network under normal condition

Figure 5: Highway network with roadworks on the dual 2-lane carriageway: (a) Exiting traffic flow through the network; (b) Total queue within the network

Figure 6: Highway network with roadworks on the single carriageway: (a) Exiting traffic flow through the network; (b) Total queue within the network

Figure 7: Highway network with roadworks on the motorway: (a) Exiting traffic flow through the network; (b) total queue within the network

\section{Table captions}

Table 1: Junction types in NTFM

Table 2: Default road capacities

Table 3: Characteristics of traffic data

Table 4 Example 1 of traffic data

Table 5 Example 2 of traffic data

Table 6: Traffic condition states for the congested junctions/links in the example network

Table 7: Road geometries for roadworks on dual 2-lane carriageway

Table 8: Road geometries for roadworks on the single carriageway 